\title{
Clinical Features and Treatment Protocol in Eleven Chinese Children with Mild COVID-19
}

\author{
Youfang Gao ${ }^{1} \cdot$ Dongfeng Zhang ${ }^{1} \cdot$ Sumin Sui ${ }^{2} \cdot$ Renying $\mathrm{Xu}^{3}$ (D)
}

Received: 30 April 2020 / Accepted: 13 May 2020 / Published online: 4 June 2020

(C) Dr. K C Chaudhuri Foundation 2020

To the Editor: Although children account for a very small proportion $(\approx 1 \%)$ among patients with COVID-19, they might also suffer from death [1]. Data regarding intervention and the outcome is limited $[2,3]$. We thus performed the current retrospective observational study involving hospitalized children in Bozhou City, Anhui Province, China.

All eleven pediatric patients (six boys and five girls, aged $10.3 \pm 3.6 \mathrm{y}$ ) with COVID-19 were recruited from our hospital from January 31 through March 2, 2020. The study protocol was approved by the Ethics Committee of the hospital.

Eight children had a close contact with suspected persons and two children were infected because of infected family members. In just one child, the exposure could not be identified. Two patients had fever, cough, and fatigue while one patient had cough. The remaining children were free of any symptoms. Virus pneumonia was confirmed by CT scan in two of the three children with clinical symptoms and three of the eight children without any symptom.

Inhaled alpha-interferon were implemented in seven children and four children received arbidol tablet because of intolerance. No antibiotics, chloroquine, and immunoglobin were implemented. A cocktail of Chinese herb was given to five children with CT-confirmed virus pneumonia. The medium time for turning to negative nucleic acid results was $9 \mathrm{~d}$ (4 to $12 \mathrm{~d})$. All the children were discharged from the hospital after a median time of $14 \mathrm{~d}$ ( 6 to $23 \mathrm{~d}$ ).

This study showed that a close contact with a suspected individual was the main reason for children's infection. Reducing the

Youfang Gao

bzgyf301@163.com

1 Department of Infection, Bozhou People's Hospital, Bozhou 236800, Anhui Province, China

2 Department of Pediatrics, Bozhou People's Hospital, Bozhou, Anhui Province, China

3 Department of Clinical Nutrition, Ren Ji Hospital, School of Medicine, Shanghai Jiao Tong University, Shanghai, China potential exposure should be recommended as a reasonable way to curb the epidemic of the disease in children. Interferon was the first recommended anti-virus treatment. In case of intolerance, arbitol could serve as an alternative. However, the necessity of anti-virus treatment needs to be further evaluated because previous studies have indicated that there are no obvious differences between children with and without anti-virus treatment $[4,5]$. Further, a cocktail of Chinese herb might be helpful to facilitate recovery. However, the results should be interpreted with caution because of small sample size.

\section{Compliance with Ethical Statements}

Conflict of Interest None.

Ethical Approval The study protocol was approved by the Ethics Committee of Bozhou People's Hospital.

Informed Consent As it was a retrospective observational study, the informed consent forms by children and their guardians were waived by the committee.

\section{References}

1. Zhu H, Wang L, Fang C, et al. Clinical analysis of 10 neonates born to mothers with 2019-nCoV pneumonia. Transl Pediatr. 2020;9:51-60.

2. Liu W, Zhang Q, Chen J, et al. Detection of Covid-19 in children in early January 2020 in Wuhan, China. N Engl J Med. 2020;382: 1370-1. https://doi.org/10.1056/NEJMc2003717.

3. Lu X, Zhang L, Du H, et al. SARS-CoV-2 infection in children. N Engl J Med. 2020;382:1663-5. https://doi.org/10.1056/ NEJMc2005073.

4. Wang XF, Yuan J, Zheng YJ, et al. [Retracted: Clinical and epidemiological characteristics of 34 children with 2019 novel coronavirus infection in Shenzhen]. Zhonghua Er Ke Za Zhi. 2020;58:E008

5. Ji LN, Chao S, Wang YJ, et al. Clinical features of pediatric patients with COVID-19: A report of two family cluster cases. World J Pediatr. 2020. https://doi.org/10.1007/s12519-020-00356-2.

Publisher's Note Springer Nature remains neutral with regard to jurisdictional claims in published maps and institutional affiliations. 